www.nature.com/jhg

\title{
Association study of the polymorphisms on chromosome 12 p13 with atherothrombotic stroke in the Japanese population
}

\author{
Tomonaga Matsushita ${ }^{1,2}$, Junji Umeno ${ }^{1,2}$, Yoichiro Hirakawa ${ }^{3}$, Koji Yonemoto ${ }^{3}$, Kyota Ashikawa ${ }^{1}$, \\ Hanae Amitani ${ }^{1}$, Toshiharu Ninomiya ${ }^{3}$, Jun $\mathrm{Hata}^{3}$, Yasufumi Doi ${ }^{3}$, Takanari Kitazono ${ }^{2}$, Mitsuo Iida ${ }^{2}$, \\ Yusuke Nakamura $^{4}$, Yutaka Kiyohara ${ }^{3}$ and Michiaki Kubo ${ }^{1,2,3}$
}

Recent genome-wide association study using four prospective population-based cohorts identified two single-nucleotide polymorphisms (SNPs) on chromosome 12p13, rs12425791 and rs11833579, to be significantly associated with the incidence of atherothrombotic stroke. To examine the association of these SNPs with atherothrombotic stroke in the Japanese population, we carried out a case-control association study using a total of 3784 cases and 3102 controls. We also examined the effect of these SNPs on the subtypes of ischemic stroke. Association analysis was carried out using logistic regression model after adjustment of age, sex and cardiovascular risk factors. Rs12425791 was significantly associated with atherothrombotic stroke $(P=0.0084$, odds ratio $(O R)=1.15)$. When we analyzed effects of rs 12425791 on ischemic stroke subtypes, rs 12425791 was significantly associated with both small-artery occlusion $(P=0.015,0 R=1.15)$ and large-artery atherosclerosis $(P=0.024$, $\mathrm{OR}=1.19)$. Rs11833579 showed no association with atherothrombotic stroke or its subtypes in our population. Our data suggest that rs 12425791 on chromosome 12 p13 is a genetic marker for atherothrombotic stroke in multiethnic population. Journal of Human Genetics (2010) 55, 473-476; doi:10.1038/jhg.2010.45; published online 7 May 2010

Keywords: atherothrombotic stroke; replication study; SNP

\section{INTRODUCTION}

Genome-wide association study (GWAS) has emerged as a powerful new approach to identify many susceptibility variants with moderate genetic risk on various common diseases, such as diabetes ${ }^{1}$ and coronary heart diseases. ${ }^{2,3}$ As for ischemic stroke, few GWASs have been carried out and genetic components of common forms of ischemic stroke are still largely unknown. Recently, the Cohorts for Heart and Aging Research in Genomic Epidemiology (CHARGE) consortium reported two single-nucleotide polymorphisms (SNPs), rs11833579 and rs12425791, to be significantly associated with the incidence of ischemic stroke in a GWAS of four population-based cohorts, which included 19602 white persons with an average of 11 years of follow-up data. ${ }^{4}$ These SNPs were located in close proximity to ninjurin 2 (NINJ2) gene on chromosome 12p13. Both SNPs showed genome-wide significance, however, only rs12425791 was replicated in both the African-American cohort and the white casecontrol sample. ${ }^{4}$ Although this study has an advantage of a prospective study design, these SNPs were merely the marker and the true causative variant(s) have not been identified yet. Moreover, this study did not analyze the effects of these SNPs on ischemic stroke subtypes probably because of small number of events.

As the association of these SNPs in Asian population remains unknown, we examined the association of these SNPs with atherothrombotic stroke using two Japanese case-control sets with a sufficient sample size. We also examined the effect of these SNPs on the subtypes of ischemic stroke.

\section{MATERIALS AND METHODS}

We used two independent Japanese case-control sets for this study. One casecontrol set (set-1) is consisted of 860 cases of atherothrombotic stroke and 860 age- and sex-matched controls. Details of the registration and case ascertainment were previously described. ${ }^{5}$ We selected 860 cases of atherothrombotic stroke on the basis of the classification as in the CHARGE study ${ }^{4}$ and subdivided them into 491 small-artery occlusion (SAO) and 369 large-artery atherosclerosis (LAA) according to the TOAST criteria. ${ }^{6}$ Age- (within 5 years) and sex-matched controls were selected from the 3196 participants of the

${ }^{1}$ Laboratory for Genotyping Development, Center for Genomic Medicine, RIKEN Yokohama Institute, Kanagawa, Japan; ${ }^{2}$ Department of Clinical Sciences, Graduate School of Medical Sciences, Kyushu University, Fukuoka, Japan; ${ }^{3}$ Department of Environmental Medicine, Graduate School of Medical Sciences, Kyushu University, Fukuoka, Japan and ${ }^{4}$ Laboratory for Molecular Medicine, Human Genome Center, The Institute of Medical Science, University of Tokyo, Tokyo, Japan

Correspondence: Dr T Matsushita, Laboratory for Genotyping Development, Center for Genomic Medicine, RIKEN, 1-7-22, Suehiro-cho, Tsurumi, Yokohama, Kanagawa 230-0045, Japan. 
Hisayama screening survey between 2002 and 2003. Another case-control set (set-2) consisted of 2924 atherothrombotic stroke and 2242 controls. Cases were selected from the BioBank Japan Project ${ }^{7}$ based on the similar criteria as in the set- 1 cases. These cases were classified into 2256 SAO and 668 LAA. The Hisayama participants who did not have ischemic stroke and did not enrolled in the set-1 were used as controls in the set-2. Clinical characteristics of the study populations are shown in Table 1.

Written informed consent was obtained from all study subjects, and this study was approved by the ethics committees of the Graduate School of Medical Sciences, Kyushu University and RIKEN Yokohama Institute.

We genotyped SNPs using the multiplex PCR-based Invader assay (Third Wave Technologies, Madison, WI, USA). ${ }^{8}$ Crude association analysis was carried out using $\chi^{2}$-test under allele model. We also assessed the association after adjustment of age, sex, body mass index, hypertension (yes/no), diabetes (yes/no) and dyslipidemia (yes/no) using logistic regression analysis under additive model. In a combined analysis, pooled estimates of the odds ratio (OR) for two case-control sets were obtained using inverse-varianceweighting analysis. ${ }^{4}$ Heterogeneities across the population were estimated formally using Cochran's Q-test. ${ }^{9}$

\section{RESULTS}

We carried out association analysis for atherothrombotic stroke using two case-control sets (Table 2). In the crude analysis, we found a weak association of rs12425791 with atherothrombotic stroke in the combined sample $(P=0.041)$, although each case-control set did not show significant association. This association became stronger after adjusted for various cardiovascular risk factors $(P=0.0084, \mathrm{OR}=1.15$, $95 \%$ confidence interval $=1.04-1.27)$. In contrast, rs 11833579 showed no association with atherothrombotic stroke even in the combined sample set $(P=0.58)$.

When we examined these associations by ischemic stroke subtypes, rs12425791 showed no association with SAO $(P=0.072)$ or LAA $(P=0.13)$ in the crude analysis. However, after adjustment of cardiovascular risk factors, rs 12425791 was significantly associated with SAO $(P=0.015, \mathrm{OR}=1.15,95 \%$ confidence interval $=1.03-1.28)$ and LAA $(P=0.024, \mathrm{OR}=1.19,95 \%$ confidence interval $=1.02-1.39)$ in the combined sample set (Table 3 ). We found no significant association of rs11833579 with either SAO or LAA.

We also carried out the association analysis stratified by sex. After adjustment of cardiovascular risk factors, rs12425791 did not show significant association with atherothrombotic stroke in men $(P=0.086$, $\mathrm{OR}=1.14)$, whereas it showed a weak association in women $(P=0.027$, $\mathrm{OR}=1.17)$. When we examined these associations by ischemic stroke subtypes, rs12425791 was associated with $\mathrm{SAO}(P=0.022, \mathrm{OR}=1.19)$, but not with LAA $(P=0.080, \mathrm{OR}=1.25)$, in women. Rs $12425791 \mathrm{did}$ not show any association with SAO $(P=0.19, \mathrm{OR}=1.11)$ or LAA $(P=0.075, \mathrm{OR}=1.20)$ in men. Rs1 1833579 showed no association with

Table 1 Clinical characteristics of the study population

\begin{tabular}{|c|c|c|c|c|}
\hline & \multicolumn{2}{|c|}{ Set-1 } & \multicolumn{2}{|c|}{ Set-2 } \\
\hline & Case & Control & Case & Control \\
\hline Male sex (\%) & 60.7 & 60.7 & 64.0 & 36.3 \\
\hline Age (years) & $70.3 \pm 9.9$ & $70.2 \pm 10.0$ & $69.1 \pm 9.2$ & $58.2 \pm 11.7$ \\
\hline Body mass index $\left(\mathrm{kg} \mathrm{m}^{-2}\right)$ & $22.0 \pm 3.9$ & $22.7 \pm 3.3$ & $23.5 \pm 3.4$ & $23.2 \pm 3.4$ \\
\hline Small-artery occlusion & 491 & & 2256 & \\
\hline Large-artery atherosclerosis & 369 & & 668 & \\
\hline \multicolumn{5}{|l|}{ Cardiovascular risk factors } \\
\hline Hypertension (\%) & 82.6 & 53.8 & 72.2 & 39.8 \\
\hline Diabetes mellitus (\%) & 32.8 & 20.6 & 15.6 & 16.2 \\
\hline
\end{tabular}

Data are shown in mean \pm s.d. or percentage except for ischemic stroke subtypes.

Table 2 Association between the SNPs reported in the CHARGE study and atherothrombotic stroke among Japanese

\begin{tabular}{|c|c|c|c|c|c|c|c|c|c|c|c|c|c|c|c|c|c|}
\hline \multirow[b]{2}{*}{ SNP (allele 1/2) } & \multirow[b]{2}{*}{ Set } & \multicolumn{5}{|c|}{ Case } & \multicolumn{5}{|c|}{ Control } & \multicolumn{3}{|c|}{ Crude } & \multicolumn{3}{|c|}{ Adjusted } \\
\hline & & 11 & 12 & 22 & Total & $M A F$ & 11 & 12 & 22 & Total & $M A F$ & P-value & $O R$ & $(95 \% \mathrm{Cl})$ & P-value & $O R$ & $(95 \% \mathrm{Cl})$ \\
\hline \multirow[t]{3}{*}{ rs12425791 (G/A) } & Set-1 & 342 & 419 & 93 & 854 & 0.35 & 392 & 360 & 107 & 859 & 0.33 & 0.22 & 1.09 & $(0.95-1.26)$ & 0.69 & 1.07 & $(0.76-1.51)$ \\
\hline & Set-2 & 1200 & 1353 & 361 & 2914 & 0.36 & 976 & 999 & 262 & 2237 & 0.34 & 0.099 & 1.07 & $(0.99-1.16)$ & 0.0084 & 1.15 & (1.04-1.28) \\
\hline & Combined & & & & & & & & & & & 0.041 & 1.08 & $(1.00-1.16)$ & 0.0084 & 1.15 & (1.04-1.27) \\
\hline \multirow[t]{3}{*}{ rs1 $1833579(G / A)$} & Set-1 & 264 & 455 & 136 & 855 & 0.43 & 292 & 422 & 146 & 860 & 0.42 & 0.55 & 1.04 & $(0.91-1.19)$ & 0.98 & 1.00 & $(0.71-1.40)$ \\
\hline & Set-2 & 942 & 1469 & 507 & 2918 & 0.43 & 749 & 1082 & 403 & 2234 & 0.42 & 0.77 & 1.01 & (0.94-1.09) & 0.58 & 1.03 & $(0.93-1.14)$ \\
\hline & Combined & & & & & & & & & & & 0.58 & 1.02 & $(0.95-1.09)$ & 0.60 & 1.03 & $(0.93-1.13)$ \\
\hline
\end{tabular}

Abbreviations: CHARGE study, the Cohorts for Heart and Aging Research in Genomic Epidemiology study; CI, confidence interval; MAF, minor allele frequency; OR, odds ratio; SNP, singlenucleotide polymorphism.

Alleles for the SNPs on the forward strand of the human genome reference sequence (NCBI build 36.3) are shown. Crude analysis was carried out using $\chi^{2}$-test under allele model. Adjusted analysis was carried out using logistic regression model after adjustment of cardiovascular risk factors. 
Table 3 Association between the SNPs reported in the CHARGE study and the subtypes of ischemic stroke among Japanese

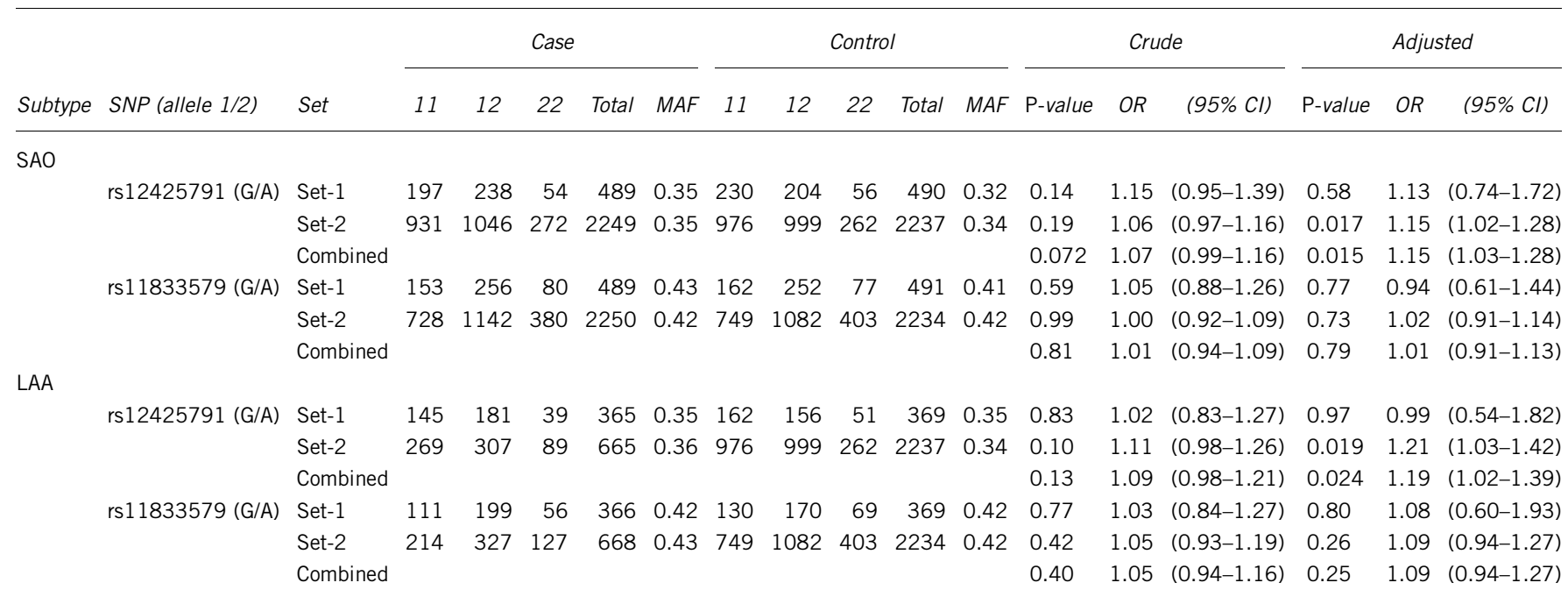

Abbreviations: CHARGE study, the Cohorts for Heart and Aging Research in Genomic Epidemiology study; CI, confidence interval; MAF, minor allele frequency; OR, odds ratio; SAO, small-artery occlusion; LAA, large-artery atherosclerosis; SNP, single-nucleotide polymorphism.

Alleles for the SNPS on the forward strand of the human genome reference sequence (NCBI build 36.3) are shown. Crude analysis was carried out using $\chi^{2}$-test under allele model.

Adjusted analysis was carried out using logistic regression model after adjustment of cardiovascular risk factors.

atherothrombotic stroke or ischemic stroke subtypes in both sexes (data not shown).

\section{DISCUSSION}

Using two independent Japanese case-control sets, we examined the association of two SNPs on chromosome 12p13 recently identified by a Caucasian GWAS of stroke. Rs12425791 was significantly associated with atherothrombotic stroke, whereas rs11833579 was not. Rs12425791 was also associated with both SAO and LAA, and its effect on the risk of SAO and LAA were similar. These results suggest that rs12425791 is a genetic marker for the incidence of atherothrombotic stroke in multiethnic populations including Japanese and might equally affect the risk of both SAO and LAA.

Similar ORs of rs12425791 on both SAO and LAA indicate that this SNP may be a marker for common pathogenesis of both ischemic stroke subtypes, probably for atherosclerosis. Rs12425791 is located at $\sim 10 \mathrm{~kb}$ proximal from the $5^{\prime}$ untranslated region of the NINJ2 gene. On the basis of the Hapmap JPT data, rs12425791 is linked to the promoter region of NINJ2. Although fine mapping is needed, SNPs linked to rs12425791 might regulate the expression level of NINJ2. Ninjurin2, a gene product of NINJ2, is a cell surface adhesion molecule and is highly expressed in the bone marrow, peripheral leukocyte, lung and lymph node in human. ${ }^{10}$ Although ninjurin2 is reported to be upregulated after nerve injury in Schwann cells and promotes neurite outgrowth, ${ }^{10}$ the function of ninjurin 2 on the ischemic stroke is largely unknown. Further functional studies are needed to clarify this issue.

Assuming the sample size of our study population using the allele frequencies in our controls and the hazard ratios in the CHARGE study, the statistical power to detect the associations at a significance level of 0.05 would be $>99 \%$ for both SNPs. However, we found a significant association of atherothrombotic stroke only in rs12425791. Similarly, the CHARGE consortium showed that the association of ischemic stroke for rs12425791 was replicated in the African-American cohort, but the association for rs11833579 was not significant. This might be due to the difference in the linkage disequilibrium between the two SNPs and true causative variant among different populations.
On the basis of the Hapmap data, linkage disequilibrium between the two SNPs was different among populations $\left(r^{2}=0.69\right.$ for JPT, $r^{2}=0.34$ for YRI and $r^{2}=0.75$ for CEU). There is a possibility that rs 12425791 is closely linked to the true causative variant of atherothrombotic stroke among different populations. In contrast, the linkage disequilibrium between true causative variant and rs11833579 will be strong in Caucasian population, but it may be weak in Japanese and AfricanAmerican populations.

The association between rs12425791 and ischemic stroke in this study was much weaker than that in the CHARGE study. The relationship of rs12425791 with atherothrombotic stroke or stroke subtypes was observed in the case-control set-2 but not in the casecontrol set-1. Furthermore, the relationship of rs12425791 with atherothrombotic stroke or stroke subtypes in the set- 2 was not detected by the $\chi^{2}$-test of allele frequencies. These results suggest that the impact of rs12425791 to atherothrombotic stroke or stroke subtypes in Japanese individuals is relatively low as compared with Caucasian population. Another possible explanation is that the effect size obtained from GWAS overestimates the true effect (winner's course). Indeed, CHARGE study showed that the genetic risk of rs12425791 in the replication study is lower than that in GWAS: in the GWAS, rs12425791 showed the strong association with atherothrombotic stroke $\left(P=3.3 \times 10^{-8}\right.$, hazard ratio=1.37), whereas it showed the $P$-value of 0.0052 and OR of 1.29 in the Dutch casecontrol study using 652 cases and 3613 controls.

In conclusion, our study suggests that rs12425791 or linked variations would be the true causative variant(s) for the genetic risk of atherothrombotic stroke in multiethnic population.

\section{CONFLICT OF INTEREST}

The authors declare no conflict of interest.

\section{ACKNOWLEDGEMENTS}

We thank the residents of Hisayama town and the patients with ischemic stroke for their participation; T Omae and the staff of the Division of Health and Welfare of Hisayama for their cooperation; many members of the Hisayama study for assistance; T Ago, H Ooboshi, M Kamouchi, H Sugimori, J Kuroda, 
Y Kumai, N Hagiwara, S Yoshimura (Kyushu University Hospital), K Tamaki, Y Wakugawa (Hakujyuji Hospital), K Fujii (Fukuoka Red Cross Hospital), Y Okada, K Toyoda (National Hospital Organization, Kyushu Medical Center), T Nagao (Imazu Red Cross Hospital), H Nakane (National Hospital Organization, Fukuoka Higashi Medical Center), S Ibayashi, Y Yamashita, K Kusuda (Seiai Rehabilitation Hospital) for clinical sample collection. We thank all the patients who participated in BioBank Japan project. We also thank all members of BioBank Japan, Institute of Medical Science, the University of Tokyo, and of the Center for Genomic Medicine, RIKEN Yokohama Institute, for their contribution to the completion of our study. This work was supported in part by the Ministry of Education, Culture, Sports, Science and Technology, Japan.

1 Wellcome Trust Case Control Consortium. Genome-wide association study of 14,000 cases of seven common diseases and 3,000 shared controls. Nature 447, 661-678 (2007).

2 Myocardial Infarction Genetics Consortium. Genome-wide association of early-onset myocardial infarction with single nucleotide polymorphisms and copy number variants. Nat. Genet. 41, 334-341 (2009).
3 McPherson, R., Pertsemlidis, A., Kavaslar, N., Stewart, A., Roberts, R., Cox, D. R. et al. A common allele on chromosome 9 associated with coronary heart disease. Science 316, 1488-1491 (2007).

4 Ikram, M. A., Seshadri, S., Bis, J. C., Fornage, M., DeStefano, A. L., Aulchenko, Y. S. et al. Genomewide association studies of stroke. N. Engl. J. Med. 360, 1718-1728 (2009).

5 Kubo, M., Hata, J., Ninomiya, T., Matsuda, K., Yonemoto, K., Nakano, T. et al. A nonsynonymous SNP in PRKCH (protein kinase $\mathrm{C} \eta$ ) increases the risk of cerebral infarction. Nat. Genet. 39, 212-217 (2007).

6 Adams, H. P. Jr. Bendixen, B. H. Kappelle, L. J., Biller, J., Love, B. B., Gordon, D. L. et al. Classification of subtype of acute ischemic stroke. Definitions for use in a multicenter clinical trial. TOAST. Trial of Org 10172 in Acute Stroke Treatment. Stroke 24, 35-41 (1993).

7 Nakamura, Y. The BioBank Japan Project. Clin. Adv. Hematol. Oncol. 5, 696-697 (2007).

8 Ohnishi, Y., Tanaka, T., Ozaki, K., Yamada, R., Suzuki, H. \& Nakamura, Y. A highthroughput SNP typing system for genome-wide association studies. J. Hum. Genet. 46, 471-478 (2001)

9 Li, D., Collier, D. A. \& He, L. Meta-analysis shows strong positive association of the neuregulin 1 (NRG1) gene with schizophrenia. Hum. Mol. Genet. 15, 1995-2002 (2006).

10 Araki, T. \& Milbrandt, J. Ninjurin2, a novel homophilic adhesion molecule, is expressed in mature sensory and enteric neurons and promotes neurite outgrowth. J. Neurosci. 20, 187-195 (2000). 